\title{
Development of the Multiplex PCR for Detection of the DNA-Contained Emergent Diseases Agents in Pigs (African Swine Fever, Aujeszky Disease, Circoviral Disease)
}

\author{
Anton Gerilovych*, Prof. Borys Stegniy, Prof. Valeriy Golovko, Dr Oleksii Solodiankin, Dr \\ Iryna Gerilovych, Yevgenia Smolyaninova and Natalya Rudova
}

NSC IECVM, Kharkiv, Ukraine

\section{Objective}

The objective was to develope the multiplex PCR for detection of the DNA-contained emergent diseases agents in pigs (African swine fever, Aujeszky disease, Circoviral disease) for diseases surveilance in pig farms

\section{Introduction}

African swine fever virus (ASFV), pseudorabies virus (Aujrszky's disease virus, $\mathrm{ADV}$ ) and porcine circovirus type $2(\mathrm{PCV}-2)$ are some of the most dangerous DNA viruses causing high amounts of morbidity and mortality in commercial and backyard pig farms. Traditional diagnosis of porcine viral infections requires complex and lengthy combinations of virological and serological tests. This study aimed to develop a method for rapid detection of the DNA-containing viral pathogens of pig in clinical materials using conventional multiplex PCR platform.

\section{Methods}

A PCR-based detection technique for ASFV, ADV and PCV-2 detection has been developed and tested under Office International Epizootical (O.I.E.) requirements, using determination of specificity, sensitivity and repeatability.

\section{Results}

The optimal conditions for amplification of ASFV, ADV and PCV-2 genome in p72, gE and cap genes, respectively, were determined. The developed protocol allowed to detect ASFV DNA by 276 bp fragment, Aujeszky disease virus and PCV-2 - 230 and 421 bp, respectively. Validation testing was performed on the panel selected agents: ASFV recombinant DNA, cloned in pUC19, ADV strain UNDIEV18V, and PCV isolates 1-02, 6-12, and 4-12. The protocol demonstrated specificity in its ability to distinguishbetween Porcine parvovirus, Classical swine fever virus, and Porcine Respiratory and Reproductive Syndrome virus. The protocol was $90 \%$ sensitive, 100 $\%$ specific and repeatable. It was recommended for validation and implementation in the laboratory practice in Ukraine.

\section{Conclusions}

A multiplex PCR platform for detection of the emergent disease agents in pigs (African swine fever, Aujeszky disease, Circoviral disease) has been developed for preliminary diagnostics of these diseases.

\section{Keywords}

multiplex PCR; porcine viroses; african swine fever 